\title{
SPECTRAL CLASSIFICATION STUDIES OF CEPHEIDS USING A SMALL TELESCOPE WITH AN IMAGE TUBE SPECTROGRAPH*
}

\author{
M. F. McCARTHY \\ Specola Vaticana, Castel Gandolfo, Vatican City State
}

\begin{abstract}
Most of the results reported thus far of image tube spectroscopy as applied in astronomy have been concerned with emission features and have been the result of a combination of the largest available light gathering power of large telescopes with the improved sensitivity of image intensifiers. We consider here the application of image tube techniques to moderate sized telescopes; concretely, we describe the first program of spectral classification using absorption features as carried out with the image tube spectrograph of the Vatican Observatory attached to the Zeiss $60-\mathrm{cm}$ reflector at Castel Gandolfo. Treanor (1970) has described the optical design, construction and the first tests of this instrument. The receiver is an RCA cascaded image tube loaned to the Vatican Observatory by the Carnegie Image Tube Committee.

The principal conclusions from the present study are the following:

(1) With sufficiently large dispersions in the grating selected, one can overcome most of the limitations of resolution inherent in the electronically formed spectral images; existing criteria for determining temperatures and luminosities can be used successfully. The dispersion used here is $95 \AA \mathrm{mm}^{-1}$; the resolution is estimated at $10 \AA$.

(2) The intensity gains of the recorded signal allow reasonable exposures to be made even with telescopes of moderate diameters; this is of special importance in the study of the spectra of variables.

(3) The absence of 'dips' in the spectral range available to the S 20 surface used in the Carnegie image tube provides many additional features for observation especially in the green-yellow-red region of the spectrum. New criteria here can complement existing criteria most of which by reasons of limited emulsion sensitivity have been limited to the spectral interval between $4000 \AA$ and $5000 \AA$.

(4) The combination of increased speed of observation and extended spectral range also facilitates one's observations of sufficient standard spectra. These can be observed very rapidly and do not constitute a block to the observation of programmed variable stars.

The objects studied here were eight Cepheids observed near maximum phase plus twenty two standard supergiant stars of types $F, G$ and $K$. The following are the Cepheids observed, SZ Tau, S Sge, SU Cyg, DT Cyg, SU Cas, FF Aql, U Aql and
\end{abstract}

* The paper in full will be published in Ric. Astron. Spec. Vat. 
$\eta \mathrm{Aql}$; they were chosen because they were relatively bright Cepheids and because abundant data for the correlation of their light and colour curves with spectral variations were available.

The limitation of observations to phases near maximum was made because both Struve (1944) and Kraft (1960) had noted that the spectral differences between variable and non-variable supergiants of late types were more pronounced near maximum phases whereas Code's suggestion (1947) was that the colours of Cepheids would be best determined at maximum phase when Cepheids of a wide range of periods had a nearly identical colour. Our observations confirm the enhancement of hydrogen and the strengthening of $\mathrm{Ti}$ as noted by the authors cited above for Cepheids at maximum as compared with non-variable standard supergiant stars of similar spectral type. The enhancement on spectra observed in this program does not seem to be of sufficient strength to cause serious blanketing problems for broad band photometry. It would be interesting on successive short exposures of the same duration to see if the enhancement of hydrogen near maximum undergoes any flickering or if it maintains a monotonic variation with phase. Another interesting point raised by the present series of observations is this: how does the enhancement of the different hydrogen lines correlate with the maximum phase which is observed to be retarded at longer wavelengths. These questions cannot be resolved from the present plate material. When the proper spectrophotometric standards can be imposed on image tube plates along with the comparison spectra and the stellar spectrum, then the use of fast image tube spectral studies can provide answers to these questions.

The preliminary attribution of features noted in spectra of late type supergiants in the interval between $4861 \AA$ and $6530 \AA$ is summarized in Table I; here the numbered feature, as observed from tracings made of the image tube spectra studied here, is given, then the approximate wavelength of the feature, its description and finally some possible sources are suggested.

TABLE I

Features observed in spectra of late-type supergiants

\begin{tabular}{|c|c|c|c|}
\hline Feature & Wavelength & Description & Suggested possible sources \\
\hline 1 & 4861 & $\mathrm{H} \beta$ & \\
\hline 2 & 5180 & Break begins & $\operatorname{MgI}(5167,5173,5184)$ \\
\hline 3 & 5300 & Line . & CrII $(5306)$; FeII $(5316)$; FeI $(5324,5328)$ \\
\hline 4 & 5505 & Break midpoint & Ca I $(5505) ; \operatorname{CrII}(5503,5508)$ \\
\hline 5 & 5680 & Break begins & ScII $(5667) ; \operatorname{ScI}(5672)$ \\
\hline 6 & 5893 & $\mathrm{NaI}$ & \\
\hline 7 & 6110 & Break begins & Ca I $(6102,6122)$ \\
\hline 8 & 6260 & Line & FeI $(6231,6254)$ \\
\hline 9 & 6350 & Break begins & FeI (6336) \\
\hline 10 & 6450 & Break begins & CaI $(6439,6462,6493)$; FeII $(6456)$ \\
\hline 11 & 6563 & $\mathrm{H} \alpha$ & \\
\hline
\end{tabular}




\section{References}

Code, A. D.: 1947, Astrophys. J. 106, 309.

Kraft, R.: 1960, Stars and Stellar Systems 6, 370.

Struve, O.: 1944, Observatory 65, 257.

Treanor, S. J., P. J.: 1970, Ric. Astron. Spec. Vat. 8, 61. 